\title{
Análise das infecções do trato respiratório em unidade de terapia intensiva de um hospital do sul do Brasil
}

\author{
Analysis of respiratory tract infections in intensive care unit of a hospital in southern Brazil \\ Análisis de infecciones del tracto respiratorio en unidad de cuidados intensivos de un hospital del
}

sur de Brasil

Recebido: 13/12/2021 | Revisado: 21/12/2021 | Aceito: 24/12/2021 | Publicado: 26/12/2021

\author{
Amanda de Souza Lemos \\ ORCID: https://orcid.org/0000-0002-8837-2823 \\ Universidade Estadual de Ponta Grossa, Brasil \\ E-mail: amanda_soule@hotmail.com \\ Laura Bazzi Longo \\ ORCID: https://orcid.org/0000-0003-1396-1524 \\ Universidade Estadual de Ponta Grossa, Brasil \\ E-mail: laurabazzilongo@hotmail.com \\ José Carlos Rebuglio Vellosa \\ ORCID: https://orcid.org/0000-0003-4747-9197 \\ Universidade Estadual de Ponta Grossa, Brasil \\ E-mail: vellosajcr@hotmail.com \\ Elisangela Gueiber Montes \\ ORCID: https://orcid.org/0000-0003-1694-085X \\ Universidade Estadual de Ponta Grossa, Brasil \\ E-mail: elisangela.gueiber@uol.com.br
}

\begin{abstract}
Resumo
As infecções hospitalares elevam a morbimortalidade e acarretam prejuízos financeiros para as instituições de saúde, sendo que o risco é maior nas unidades de terapia intensiva, devido a fragilidade dos pacientes, uso de antibióticos de largo espectro e procedimentos invasivos frequentes. Nesse contexto, o trato respiratório é o mais acometido e tem como principal fator de risco a ventilação mecânica. O presente trabalho tem como objetivo determinar a prevalência e as características das infecções respiratórias ocorridas em pacientes internados nessas unidades. Trata-se de um estudo retrospectivo, realizado a partir das notificações de infecção hospitalar, de janeiro de 2017 a dezembro de 2019, ocorridas nas unidades de terapia intensiva de um hospital terciário do Paraná. Houve 398 notificações, sendo o trato respiratório responsável por $68,3 \%$. As pneumonias associadas à ventilação mecânica foram as mais prevalentes, apesar de demonstrar um declínio no período estudado. As traqueobronquites, embora menos prevalentes, apresentaram a maior letalidade (31,6\%). O Staphylococcus aureus resistente à meticilina foi o microrganismo mais frequente (11\%), seguido de Pseudomonas aeruginosa (9,6\%). Enquanto isso, o esquema terapêutico mais utilizado para o tratamento dessas infecções foi piperacilina-tazobactam (43\%). Este estudo contribuiu com dados epidemiológicos das infecções nosocomiais da instituição pesquisada, possibilitando elencar estratégicas e medidas de prevenção com maior especificidade e direcionamento voltado à realidade local, além de poder ser utilizado para comparação em estudos futuros.
\end{abstract}

Palavras-chave: Infecção Hospitalar; Pneumonia Associada a Assistência à Saúde; Pneumonia Associada à Ventilação Mecânica.

\begin{abstract}
Hospital infections increase morbidity and mortality and cause financial losses for health institutions, and the risk is higher in intensive care units, due to patients' frailty, the use of large spectrum antibiotics and frequent invasive procedures. In this regard, respiratory tract is the most affected and its main risk factor is the mechanical ventilation. This study aims to determine prevalence and characteristics of respiratory infections that occur in patients hospitalized in these units. This is a retrospective study, based on notifications of hospital infection, from January 2017 to December 2019, which occurred in intensive care units of a tertiary hospital in Paraná. There was a total of 398 notifications, while the respiratory tract was responsible for $68.3 \%$ of the cases. Ventilator-associated pneumonias were the most prevalent. Nevertheless, it showed a decline in the period studied. Tracheobronchitis, although less prevalent, had the highest lethality rate (31.6\%). Methicillin-resistant Staphylococcus aureus was the most frequent microorganism (11\%), followed by Pseudomonas aeruginosa (9.6\%). Yet, the most used therapeutic regimen for these infections' treatment was piperacillin-tazobactam (43\%). This study contributed with epidemiological data of nosocomial infections from the
\end{abstract}


researched institution, making it possible to elect strategics and prevention measures with greater specificity and targeting the local reality. In addition, it can be useful in comparations with future studies.

Keywords: Cross Infection; Healthcare-Associated Pneumonia; Ventilator-Associated Pneumonia.

\section{Resumen}

Las infecciones hospitalarias aumentan la morbilidad y la mortalidad y causan pérdidas económicas a las instituciones de salud, siendo que el riesgo es mayor en las unidades de cuidados intensivos, debido a la fragilidad de los pacientes, el uso de antibióticos de amplio espectro y los frecuentes procedimientos invasivos. En este contexto, el tracto respiratorio es el más afectado y su principal factor de riesgo es la ventilación mecánica. Este estudio tiene como objetivo determinar la prevalencia y las características de las infecciones respiratorias que se presentan en los pacientes hospitalizados en estas unidades. Se trata de un estudio retrospectivo, basado en notificaciones de infección hospitalaria, de enero de 2017 a diciembre de 2019, ocurrida en las unidades de cuidados intensivos de un hospital terciario de Paraná. Hubo 398 notificaciones, siendo el tracto respiratorio el responsable del 68,3\%. Las neumonías asociadas al ventilador fueron las más prevalentes, a pesar de mostrar un descenso en el período estudiado. La traqueobronquitis, aunque menos prevalente, tuvo la mayor letalidad (31,6\%). Staphylococcus aureus resistente a meticilina fue el microorganismo más frecuente (11\%), seguido de Pseudomonas aeruginosa $(9,6 \%)$. Mientras tanto, el régimen terapéutico más utilizado para el tratamiento de estas infecciones fue piperacilina-tazobactam (43\%). Este estudio aportó datos epidemiológicos de infecciones nosocomiales en la institución investigada, permitiendo elegir estrategias y medidas de prevención con mayor especificidad y focalización en la realidad local, además puede ser utilizado para la comparación con estudios futuros.

Palabras clave: Infección Hospitalaria; Neumonía Asociada a la Atención Médica; Neumonía Asociada al Ventilador.

\section{Introdução}

A Infecção Hospitalar (IH) é definida, segundo a Portaria nº 2.616 de 12/05/1998 do Ministério da Saúde, como qualquer infecção adquirida após a internação do paciente e que se manifesta durante a internação ou mesmo após a alta, quando puder ser relacionada com a internação ou procedimentos hospitalares (Portaria nº 2616, 1998). Além da expressiva morbimortalidade, com riscos para os pacientes, menor qualidade de vida e hospitalizações mais longas, as infecções nosocomiais ainda acarretam custos elevados para os hospitais, o que torna sua prevenção um imperativo de gestão (Neves et al., 2019). Atualmente, o termo Infecção Hospitalar tem sido substituído por Infecção Relacionada à Assistência à Saúde (IRAS), com a finalidade de abranger não só a infecção adquirida no hospital, mas também aquela relacionada a procedimentos feitos em ambulatório, durante cuidados domiciliares e à infecção ocupacional adquirida por profissionais de saúde (Horan et al., 2008).

As IH tornam-se ainda mais relevantes em unidades de terapia intensiva (UTI), onde há um risco de 5 a 10 vezes maior de adquirir infecção, devido a vulnerabilidade dos pacientes, em estado grave e comprometidos imunologicamente, além de procedimentos invasivos frequentes e uso de antibióticos de largo espectro (Moura et al., 2007). Estima-se que neste setor ocorram aproximadamente $25 \%$ de todas as infecções nosocomiais, o que torna a UTI tema de estudos na busca de maior eficácia no tratamento das infecções e menor tendência ao aparecimento de microrganismos multirresistentes, bem como redução do tempo de internação dos pacientes e de gastos do hospital (Zaragoza et al., 2014).

No que diz respeito à localização topográfica, as infecções do trato respiratório são as mais frequentes, como é apresentado por Padrão et al. (2010) em um estudo de prevalência de infecções hospitalares em UTI, onde o trato respiratório foi o mais acometido, representando $44,19 \%$ do total. O risco de infecção respiratória é três a dez vezes maior nos pacientes sob ventilação mecânica $(\mathrm{VM})$ invasiva, sendo este o principal fator de risco, em que os pacientes ficam acamados por tempo prolongado, favorecendo o acúmulo de secreção nas vias aéreas (Leiser et al., 2007). Estudos demonstraram a prevalência de cocos gram-positivos, especialmente Staphylococcus aureus (S. aureus), como causa das infecções respiratórias nos pacientes em UTI. Além disso, verifica-se uma alta incidência de microrganismos resistentes a um ou mais fármacos antimicrobianos, principalmente gentamicina e ciprofloxacino, o que evidencia a necessidade do diagnóstico adequado e a dificuldade no tratamento destes pacientes (Calcagnotto et al., 2011).

A pneumonia é uma infecção pulmonar identificada pela utilização de uma combinação de critérios. Segundo os critérios diagnósticos de infecção relacionada à assistência à saúde da Agência Nacional de Vigilância Sanitária (ANVISA), ela pode ser 
definida clinicamente pela presença de infiltrado, opacificação ou cavitação em radiografia de tórax; associada a febre, leucopenia ou leucocitose, ou alterações no nível de consciência em pacientes mais idosos; e apresentar pelo menos dois desses sinais: alteração na secreção pulmonar, piora da troca gasosa, ausculta com roncos ou estertores, e tosse ou dispneia ou taquipneia. O diagnóstico pode ser facilitado microbiologicamente, através de culturas ou sorologias (ANVISA, 2017).

A pneumonia associada a ventilação mecânica (PAV) possui os mesmos critérios epidemiológicos, quando diagnosticada no paciente que está em ventilação mecânica por um período maior que dois dias, e que no dia do diagnóstico estava em VM ou havia removido o dispositivo no dia anterior. Atualmente, novos critérios para o diagnóstico de PAV estão sendo debatidos. Já a traqueobronquite é diagnosticada quando não há evidência clínica ou radiológica de pneumonia, enquanto apresenta pelo menos dois dos seguintes sinais e sintomas: febre, tosse, aparecimento ou aumento da secreção, roncos e sibilos. A traqueobronquite também pode ser confirmada pela cultura positiva de aspirado traqueal ou broncoscopia (ANVISA, 2017).

Em indivíduos sob terapia intensiva, o reflexo da tosse, a capacidade de expectoração e as barreiras imunológicas estão deficientes por perderem seus mecanismos de defesa, o que os torno propensos a infecções respiratórias. A aspiração de microrganismos da cavidade oral é a via de acesso mais comum, o que significa que a microbiota bucal tem papel primordial na etiologia das infecções respiratórias. O mais agravante é o fato de que, em apenas 48 horas após a entrada na UTI, os pacientes já apresentam a orofaringe colonizada. Portanto, além das medidas prevenção tradicionais, deve-se incluir nestes pacientes a higiene bucal (Barbosa et al., 2010).

Os fatores de risco para pneumonia hospitalar são idade acima de 60 anos, gênero masculino, extremos do estado nutricional, tempo de internação hospitalar prolongado, comorbidades, especialmente a doença pulmonar obstrutiva crônica, fatores associados a cirurgia, uso de sondas gástricas, entre outros. Alguns desses fatores de risco não podem ser modificados. Em contrapartida, os modificáveis podem ser controlados por meio de medidas simples, tais como: lavagem das mãos; instituição de protocolos que visem a redução de prescrições inadequadas de antimicrobianos e vigilância microbiológica, com informação periódica aos profissionais, quanto à prevalência e resistência da microbiota (C. M. dos Santos et al., 2019).

Neste contexto, é imprescindível o correto diagnóstico e avaliação da doença respiratória, o uso racional de antimicrobianos e o controle no ambiente hospitalar, evitando a disseminação de microrganismos resistentes, principalmente nas afecções respiratórias, facilmente transmissíveis entre pacientes. O conhecimento dos microrganismos e seus respectivos padrões de suscetibilidade são importantes como instrumentos de orientação para a terapêutica inicial, bem como para o estabelecimento de protocolos e ações das comissões de controle de infecções hospitalares.

Logo, visto que o perfil microbiológico e a sensibilidade aos fármacos antimicrobianos dependem de cada instituição hospitalar e podem variar entre as regiões demográficas, se faz necessária a obtenção de dados regionais. Realizar pesquisas para avaliar os principais indicadores e caracterizar as infecções é essencial para se estabelecer medidas de controle e prevenção das Infecções Hospitalares. Este é um desafio contínuo para os hospitais e profissionais de saúde, no qual o presente estudo tem o objetivo de auxiliar.

\section{Metodologia}

Trata-se de um estudo do tipo descritivo transversal, com delineamento retrospectivo, realizado por meio dos arquivos do Núcleo de Epidemiologia e Controle de Infecção Hospitalar (NUCIH) do Hospital Universitário Regional dos Campos Gerais. Este é um hospital público, de nível terciário, atende a 12 municípios do estado do Paraná, e possuía 20 leitos de UTI adulto durante o período estudado.

A coleta de dados foi realizada a partir das notificações de infecção hospitalar do NUCIH, ocorridas nas duas alas de unidade de terapia intensiva deste hospital, de janeiro de 2017 a dezembro de 2019.

Posteriormente, foram selecionadas apenas as notificações relacionadas ao trato respiratório, as quais foram submetidas 
a revisão de prontuário e análise das culturas microbiológicas. Levantou-se dados a respeito das características dos pacientes, tempo de internação, curso da doença e confirmação do agente etiológico, obtidos através do Sistema de Gestão da Assistência de Saúde do SUS (GSUS). Os dados encontrados foram processados em planilhas eletrônicas do programa Microsoft Office Excel®, para realização da análise estatística descritiva.

Quanto aos aspectos éticos, o estudo foi submetido ao Comitê de Ética em Pesquisa da referida instituição, sendo aprovado com o número de parecer 4.110.874.

\section{Resultados e Discussão}

No período de três anos analisados no presente estudo, foram notificados 398 casos de IRAS, com uma média anual de 132,7. Neste mesmo período, ocorreram 2.992 internações nas unidades de terapia intensiva do hospital, resultando em uma taxa de IH de 13,3\%. O número de notificações apresentou uma queda ao longo dos anos, passando de uma taxa de 18,2\% (174 notificações) em 2017, para 6,9\% (72) em 2019.

Os valores encontrados estão dentro do esperado quando comparado com outras instituições. O estudo realizado por Leiser et al. (2007) mostrou como resultado uma taxa de IH de 15,95\% e demonstrou a ocorrência de infecção nosocomial em UTI em 208 pacientes no período de um ano, enquanto a pesquisa de R. P. Santos et al. (2014) apresentou 96 registros no mesmo período, com uma taxa de $27,35 \%$. Ademais, uma análise anterior avaliou as infecções hospitalares neste mesmo hospital no ano de 2007, e notificou a ocorrência de 135 casos na UTI adulto, semelhante ao que foi encontrado agora (Gaspar et al., 2012). Entretanto, a comparação de valores em percentuais para taxas de IRAS deve ser realizada com cautela, visto que são números muito específicos de cada instituição. A metodologia adotada na investigação, o tipo do estabelecimento e a gravidade de seus atendimentos, condições tecnológicas, capacitação técnica da equipe, o tempo de internação dos pacientes, entre outras, são variáveis que devem ser rigorosamente consideradas ao se analisar esse tipo de resultados (Leiser et al., 2007).

Dentre as infecções hospitalares em UTI, o trato respiratório foi o mais acometido, apresentando 272 notificações, sendo, portanto, o enfoque deste estudo. Quanto ao perfil demográfico desses pacientes (Tabela 1), a maioria eram homens (69,5\%) e a idade variou de 17 a 96 anos, com uma média de 56,9 ( $\pm 18,7)$. O tempo médio de internação foi de 33,3 dias ( \pm 23,7), sugerindo a relação entre internações mais longas e IH. A literatura descreve que o tempo de permanência aumenta diante da presença de infecção hospitalar e também é fator de risco para ela, além de demonstrar que as pneumonias nosocomiais são uma das principais complicações relacionadas ao prolongamento dos internamentos (Rufino et al., 2012; Sinésio et al., 2018). Ademais, outro fator fortemente associado a ocorrência de infecções hospitalares em trato respiratório é o uso de ventilação mecânica. Foi observado que apenas 4 pacientes não foram submetidos a este dispositivo invasivo durante o internamento, reforçando seu papel nestas afecções. 
Tabela 1 - Caracterização dos pacientes com infecções respiratórias.

\begin{tabular}{llll}
\hline \multirow{2}{*}{ Sexo } & Variável & n & \% \\
\hline & Feminino & 83 & $30,5 \%$ \\
\cline { 2 - 4 } & Masculino & 189 & $69,5 \%$ \\
\hline \multirow{3}{*}{ Faixa etária } & $<20$ & 6 & $2,2 \%$ \\
\cline { 2 - 4 } & $20-39$ & 49 & $18 \%$ \\
\cline { 2 - 4 } & $40-59$ & 78 & $28,7 \%$ \\
\cline { 2 - 4 } & $60-79$ & 113 & $41,5 \%$ \\
\cline { 2 - 4 } Ventilação Mecânica & $\geq 80$ & 26 & $9,6 \%$ \\
\hline \multirow{2}{*}{$\begin{array}{l}\text { Tempo de internação } \\
\text { (em dias) }\end{array}$} & Sim & 4 & $1,5 \%$ \\
\cline { 2 - 4 } & Não & 268 & $98,5 \%$ \\
\hline \multirow{2}{*}{ Desfecho final } & $15-30$ & 116 & $15,4 \%$ \\
\cline { 2 - 4 } & $>30$ & 114 & $41,9 \%$ \\
\hline
\end{tabular}

Fonte: Autores.

A alta prevalência de infecções respiratórias de $68,3 \%$ foi seguida por infecções do trato urinário e infecções primárias da corrente sanguínea. Condizente aos resultados deste estudo, para Padrão et al. (2010), o trato respiratório também foi o mais acometido, bem como para Leiser et al. (2007), em que a pneumonia foi a infecção mais frequente, alcançando 66,34\%. R. P. Santos et al. (2014) concorda que este é o sítio mais prevalente, no entanto, com um percentual menor, de 36,46\%. O gráfico 1 resume a distribuição dos tipos de infecções nosocomiais em UTI.

Gráfico 1 - Infecções Hospitalares em UTI, 2017 a 2019.

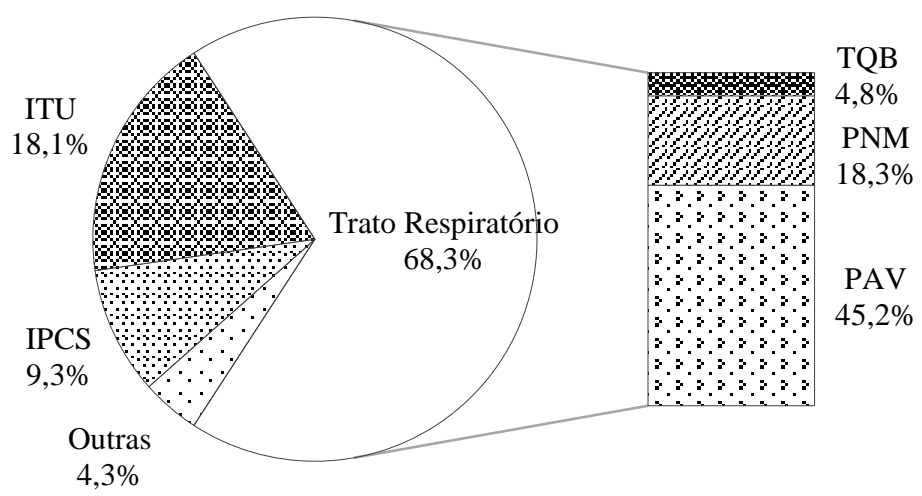

IPCS - infecção primária de corrente sanguínea; ITU - infecção do trato urinário; PAV - pneumonia associada a ventilação mecânica; PNM - pneumonia nosocomial; TQB - traqueobronquite. Fonte: Autores.

Mundialmente, esse cenário se repete, como é visto no estudo de prevalência europeia de infecção em terapia intensiva (EPIC), que demonstra que as infecções pulmonares são as mais prevalentes em cuidados críticos, além de associar a pneumonia adquirida em UTI com maior risco de mortalidade. Na Espanha, o estudo nacional de vigilância de infecção hospitalar em UTI (ENVIN-UCI) documentaram a pneumonia associada a ventilação mecânica como a mais frequente, mas destacando o 
decréscimo nas taxas desta patologia ao longo dos anos. Assim como o sistema de vigilância nacional belga, o qual demonstrou uma taxa de 12 episódios de PAV por 1.000 dias de ventilação mecânica (Zaragoza et al., 2014).

De acordo com o NUCIH, as notificações de infecções respiratórias foram classificadas em pneumonia associada à ventilação mecânica (PAV), pneumonia nosocomial (PNM) e traqueobronquite (TQB). A primeira foi a mais prevalente $(66,2 \%)$, com 180 casos ao total. Estudos mostram que o risco relativo associado a ventilação mecânica é de 2,57 (Leiser et al., 2007), corroborando com a alta prevalência de PAV encontrada. Por outro lado, notou-se um decréscimo no número de PAV no período estudado, sendo que a traqueobronquite se torna a mais prevalente no último ano. Foram notificados em 2017, 2018 e 2019 , respectivamente, 98, 68 e 14 casos de PAV; 29, 30 e 14 de PNM; e 0, 2 e 17 de TQB (Gráfico 2).

Gráfico 2 - Infecções Hospitalares do Trato Respiratório por ano.

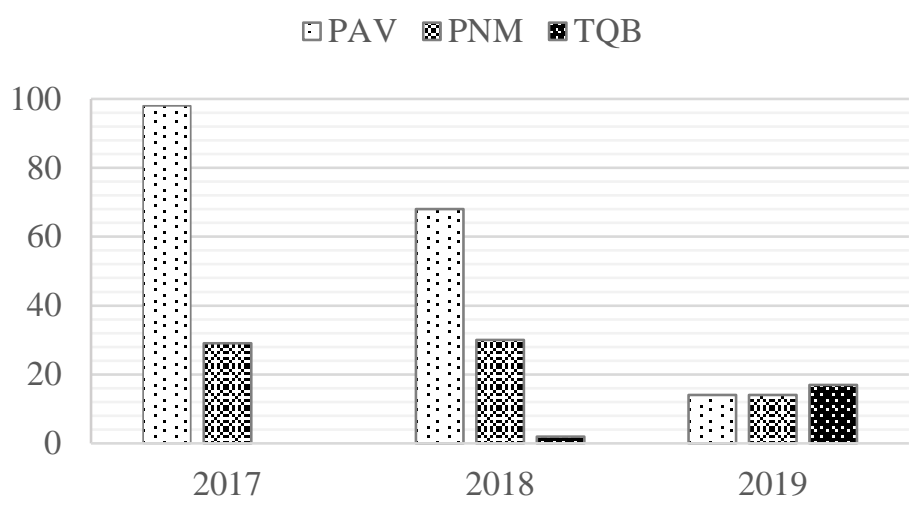

PAV - pneumonia associada a ventilação mecânica; PNM - pneumonia nosocomial; TQB - traqueobronquite. Fonte: Autores.

Artigos de revisão demonstram que as incidências de PAV variam amplamente de 5 a 40\%, dependendo do país, tipo de UTI e critérios diagnósticos. Por outro lado, assim como o declínio no número de PAV observado no gráfico acima, as taxas vêm reduzindo nas últimas duas décadas, devido ao maior conhecimento da fisiopatologia e, principalmente, a implementação de programas adequados de prevenção (Papazian et al., 2020). Em contrapartida, a traqueobronquite, que se diferencia pela ausência de evidência de sinais radiológicos de pneumonia, tem sido negligenciada e sua prevalência pode variar de próximo a 0\% até 15\% em pacientes em VM (Salluh et al., 2019). O aumento expressivo na incidência de traqueobronquite em 2019 encontrado neste estudo pode ser explicado pela melhora diagnóstica e ações propostas pela comissão de controle de infecção hospitalar, o que deve ser encorajado em todas as instituições. Já a pneumonia nosocomial apresenta uma incidência geral de 1,6\% em estudos desenvolvidos nos Estados Unidos, o que representa uma taxa de 3,63 por mil pacientes-dia. Apesar disso, as pneumonias não associadas a ventilação mecânica são subestimadas e pouco estudadas, visto que a maioria das pesquisas se concentram na PAV (Giuliano et al., 2018).

Quanto ao perfil microbiológico, o microrganismo mais prevalente foi Staphylococcus aureus resistente à meticilina (MRSA), sendo isolado 30 vezes (11\%). A cepa sensível de S. aureus também possui relevância e foi isolado em 8,5\% das notificações. O S. aureus é um coco gram-positivo, com elevada resistência antimicrobiana e frequentemente associado a infecções hospitalares, sendo que a proporção de MRSA ultrapassa 50\% entre os pacientes internados em UTI (Moura et al., 2007). A resistência à meticilina vem associada a outros principais antimicrobianos, limitando as opções de tratamento. No presente estudo não houve nenhum caso de resistência à vancomicina, mas esta é a adaptação genética mais temida atualmente, devido a ampla dependência desse antibiótico no tratamento de infecções por MRSA (Turner et al., 2019). Este patógeno apresenta uma elevada incidência como agente de PAV, além de estar associado a alta mortalidade, especialmente em unidades 
de terapia intensiva (Karvouniaris et al., 2011). Portanto, medidas de prevenção de infecção, incluindo triagem, isolamento de contato e boas práticas de higiene das mãos, devem ser prioritárias.

O segundo microrganismo mais prevalente foi Pseudomonas aeruginosa ( $P$. aeruginosa), responsável por 9,6\% dos casos. Este bacilo Gram-negativo não fermentador de glicose é um dos principais agentes de infecções respiratórias nas UTIs e as infecções causadas por ele estão associadas à alta letalidade, devido a limitação das opções terapêuticas. Estudos em que a $P$. aeruginosa foi identificada em $25 \%$ das culturas de secreções traqueais positivas, demonstraram que mais da metade das cepas foram resistentes aos principais antibióticos utilizados, como cefepime, ceftazidima, ciprofloxacina e meropenem (Rocha \& Dias, 2015).

Outro bacilo Gram-negativo não fermentador de glicose muito frequente em infecções hospitalares é o Acinetobacter baumannii (A. baumannii). A sobrevivência desses dois agentes em água e outros ambientes com requisição de nutrientes favorecem sua presença no trato respiratório, por colonizarem coleções de água relacionadas ao aparato de ventilação mecânica (Silva et al., 2021). No entanto, no presente estudo, A. baumannii teve uma taxa de 5,5\%, abaixo do que é encontrado em literatura. Para Leiser et al. (2007), essa bactéria apresentou uma incidência de 13,76\%, maior que a taxa de P. aeruginosa de 9,42\%. Outros estudos apresentam taxas maiores do que $25 \%$ de resistência a cefalosporinas e também apresentando elevada resistência a carbapenêmicos, que são as drogas de escolha para o tratamento de infecções por esse microrganismo (Rocha \& Dias, 2015).

As enterobactérias também estão entre os principais agentes de IH, como exemplo, Klebsiella pneumoniae $(8,1 \%)$ e Escherichia coli $(4,8 \%)$. Além disso, essa família apresenta mecanismos de resistência principalmente por meio das enzimas produtoras de betalactamases, que hidrolisam o anel betalactâmico. Dentro disso, destaca-se as betalactamases de espectro estendido (ESBL) e as carbapenemases do tipo KPC (Klebsiella pneumoniae carbapenemase) (Bush \& Bradford, 2020). Nesta pesquisa, foram identificados 11 (4\%) casos de KPC e 8 (2,9\%) de ESBL. O gráfico 3 reúne os agentes etiológicos das notificações de IH do trato respiratório encontrados neste estudo.

Gráfico 3 - Perfil microbiológico das infecções hospitalares do trato respiratório em UTI.

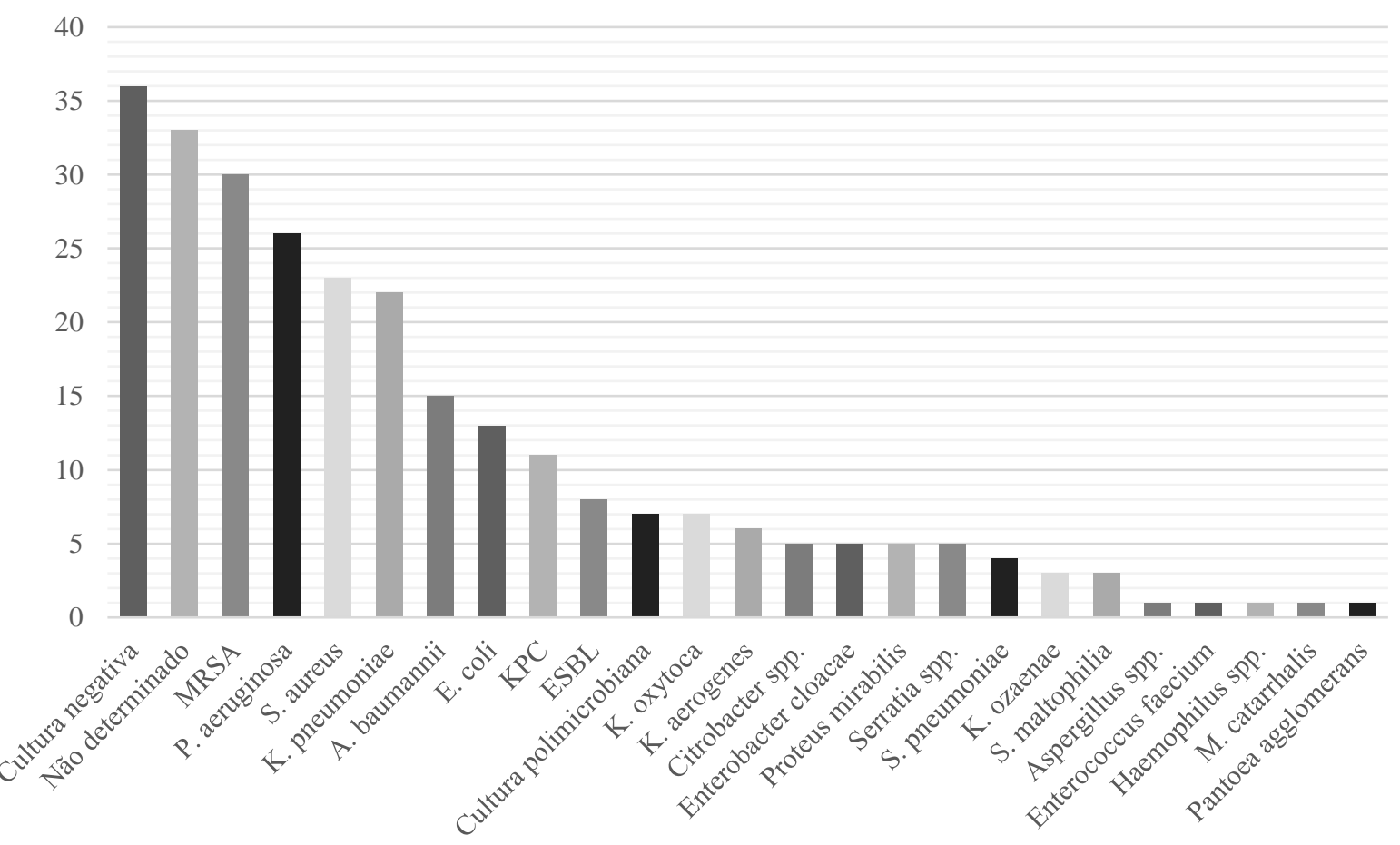


Demonstrando a gravidade do problema acerca das enterobactérias, um estudo retrospectivo realizado na China mostrou que $68,8 \%$ dos pacientes com diagnóstico de pneumonia bacteriana adquirida no hospital foram identificados com infecção por KPC, dos quais 25\% morreram em 28 dias (Pang et al., 2018). A mortalidade nos casos de ESBL também é alta, chegando a 55\% (Starzyk-Łuszcz et al., 2017), sendo que sua proporção em infecções nosocomiais tem aumentado significativamente nos últimos anos em países como a Alemanha (Leistner et al., 2015). Dentre essa família de bactérias, a Klebsiella pneumoniae possui predisposição a causar infecções nosocomiais e a habilidade de acumular e transferir mecanismos de resistência, mas, apesar disso, outras espécies também podem ser produtoras das enzimas betalactamases (Magalhães \& Soares, 2018).

A cultura polimicrobiana, também chamada mista, engloba aquelas onde houve o crescimento de mais um microrganismo. Dentre as sete culturas polimicrobianas identificadas neste estudo, encontrou-se a associação de: Enterobacter cloacae e Klebsiella aerogenes; Escherichia coli e Proteus mirabilis; ESBL e Proteus mirabilis; MRSA e Stenotrophomonas maltophilia; S. aureus e Morganella morganii; P. aeruginosa e KPC, sendo que esta última associação apareceu duas vezes.

Por fim, quase todas as infecções foram causadas por bactérias, exceto por um caso de Aspergillus spp. No entanto, em um quarto das notificações $(25,4 \%)$, o agente etiológico não foi determinado ou a cultura foi negativa. Apesar de dificultar a vigilância epidemiológica, isso também ocorre em outros estudos, como em R. P. Santos et al. (2014), onde relatou-se que 28,13\% das infecções tiveram outros agentes etiológicos como causa ou não havia identificação acerca do nome do microrganismo no registro.

A emergência e a disseminação de patógenos resistentes é propiciada por condições como as características da assistência ao paciente em UTI, associado aos esquemas terapêuticos de amplo espectro e uso de doses mais elevadas, o que ainda aumenta a suscetibilidade dos pacientes à contaminação por esses agentes. Taxas de IRAS por microrganismos multirresistentes são ainda maiores nas unidades de cuidado intensivo (Leiser et al., 2007), e as taxas de mortalidade mais elevadas estão relacionadas com o envolvimento de patógenos de alto risco ou nas situações em que a antibioticoterapia inicial não é apropriada para o agente causal (Calcagnotto et al., 2011).

As taxas de resistência variam localmente na dependência do consumo de antimicrobianos. Por isso, o uso racional de antibióticos e o uso de dados locais sobre o perfil de sensibilidade microbiana são estratégias para prevenção de infecções. Nas 272 notificações do trato respiratório, foram utilizados 29 esquemas terapêuticos diferentes como tratamento inicial, sendo que a maioria era composto pela associação de dois ou mais antibióticos. A tabela 2 representa as classes farmacológicas utilizadas. O esquema empírico mais prescrito foi piperacilina acompanhada de tazobactam, sendo amplamente utilizado em $43 \%$ dos casos. A monoterapia com meropenem $(11,4 \%)$ e o uso conjunto de ceftazidima e oxacilina $(7,7 \%)$ foram outros esquemas antimicrobianos mais frequentemente utilizados. Demonstrando que esses dados variam entre as instituições, para Calcagnotto et al. (2011), os antimicrobianos mais prescritos foram a ceftriaxona e a sultamicilina, enquanto piperacilina-tazobactam não foi utilizado nenhuma vez. Este mesmo estudo avaliou as bactérias isoladas no trato respiratório em pacientes admitidos em UTI, e relatou que apenas $24 \%$ da amostra não apresentava microrganismos resistentes a nenhum tipo de antimicrobiano (Calcagnotto et al., 2011). 
Tabela 2 - Esquemas terapêuticos utilizadas no tratamento das infecções respiratórias, por classe de medicamento.

\begin{tabular}{|c|c|c|}
\hline Esquema terapêutico & $\mathbf{n}$ & $\%$ \\
\hline Penicilina & 120 & $44,1 \%$ \\
\hline Carbapenêmico & 31 & $11,4 \%$ \\
\hline Cefalosporina & 22 & $8,1 \%$ \\
\hline Cefalosporina + Penicilina & 21 & $7,7 \%$ \\
\hline Cefalosporina + Macrolídeo & 14 & $5,1 \%$ \\
\hline Cefalosporina + Lincosamida & 13 & $4,8 \%$ \\
\hline Quinolona & 10 & $3,7 \%$ \\
\hline Glicopeptídeo + Carbapenêmico & 8 & $2,9 \%$ \\
\hline Esquema terapêutico & $\mathbf{n}$ & $\%$ \\
\hline Glicopeptídeo & 7 & $2,6 \%$ \\
\hline Aminoglicosídeo & 5 & $1,8 \%$ \\
\hline Sulfonamida & 4 & $1,5 \%$ \\
\hline Anfenicol & 3 & $1,1 \%$ \\
\hline Cefalosporina + Nitroimidazólico & 3 & $1,1 \%$ \\
\hline Tetraciclina & 2 & $0,7 \%$ \\
\hline Cefalosporina + Anfenicol & 1 & $0,4 \%$ \\
\hline Cefalosporina + Glicopeptídeo & 1 & $0,4 \%$ \\
\hline Cefalosporina + Penicilina + Nitroimidazólico & 1 & $0,4 \%$ \\
\hline Glicopeptídeo + Carbapenêmico + Equinocandina & 1 & $0,4 \%$ \\
\hline Glicopeptídeo + Sulfonamida & 1 & $0,4 \%$ \\
\hline Polimixina & 1 & $0,4 \%$ \\
\hline Polimixina + Aminoglicosídeo & 1 & $0,4 \%$ \\
\hline Quinolona + Nitroimidazólico & 1 & $0,4 \%$ \\
\hline Triazólico & 1 & $0,4 \%$ \\
\hline Total Geral & 272 & $100 \%$ \\
\hline
\end{tabular}

Fonte: Autores.

A taxa de letalidade por todas as infecções hospitalares em UTI foi de 20,9\% (83/398), e é próxima ao que foi encontrado por Leiser et al. (2007), que relataram uma taxa de 27,88\%. Outro estudo mostrou que 15,4\% dos óbitos de um hospital estavam associados à infecção hospitalar, sendo 8,7\% diretamente devido à infecção adquirida no serviço. As infecções de corrente sanguínea foram as mais prevalentes entre os óbitos, mas as pneumonias ocupam logo a segunda posição, sendo a maior parte associada a VM e em maiores de 60 anos (Guimarães et al., 2011).

Neste estudo, a taxa de letalidade por infecções nosocomiais do trato respiratório encontrada foi de 21,3\% (58/272). Apesar da menor prevalência, a traqueobronquite se mostrou mais letal, com uma taxa de 31,6\%, requisitando maior atenção. Houve ainda 43 óbitos em decorrência de pneumonias associadas à ventilação mecânica, resultando em uma taxa de letalidade de 23,9\%. Além disso, pouco mais da metade dos pacientes foram a óbito como desfecho final de sua internação, sugerindo que a IH acarreta maior mortalidade também indiretamente. Os óbitos associados à infecção hospitalar podem representar cerca de 15\% de todas as causas, e, dentre esses, as pneumonias somam quase setenta por cento (Guimarães et al., 2011). Barbosa et al. (2010) demonstrou que 35,6\% dos pacientes internados com pneumonia nosocomial evoluíram a óbito, confirmando que a taxa de letalidade das infecções respiratórias em UTI é elevada, podendo variar entre 33\% e 71\% (Moura et al., 2007).

As infecções hospitalares são consideradas importantes fatores de complicação em pacientes internados em UTI, 
contribuindo para o aumento das taxas de morbidade, de mortalidade e de tempo de permanência no hospital, além de elevar custos do tratamento. Devido a magnitude deste problema, torna-se imprescindível o papel das Comissões de Controle de Infecção Hospitalar, agindo através da vigilância epidemiológica, estabelecimento de protocolos clínicos, utilização de medidas de isolamento, de materiais e equipamentos adequados, higienização do ambiente, identificação de bactérias multirresistentes, antibioticoterapia adequada, treinamento da equipe multiprofissional, implementação de medidas de controle, entre outros fatores que podem impactar os resultados.

O presente estudo pode auxiliar no trabalho da comissão da instituição pesquisada, ao apontar os avanços que devem ser mantidos e as lacunas que devem ser melhoradas, direcionando a aplicação de ações, como as citadas anteriormente. Por outro lado, o alto percentual de casos sem identificação do agente microbiano prejudica a visualização do panorama real do contexto hospitalar estudado, necessitando de maior atenção a fatores que prejudicam o resultado das culturas microbiológicas, como a coleta inadequada e o uso anterior de antimicrobianos. Além disso, o adequado preenchimento das notificações de $\mathrm{IH}$ deve ser reforçado, a fim de fomentar informação e possibilitar resultados mais fiéis e que reflitam melhor a realidade.

\section{Conclusão}

As infecções respiratórias prevaleceram, bem como o que é relatado na literatura. Houve um predomínio da PAV, junto ao intenso uso da ventilação mecânica em UTI. O número de IH vem diminuindo ao longo dos anos, demonstrando a importância da constante vigilância e continuidade das ações de prevenção e controle.

O microrganismo mais prevalente foi o Staphylococcus aureus resistente à meticilina, o que denota ao crescente número de bactérias multirresistentes. O conhecimento de agentes bacterianos com seus respectivos padrões de suscetibilidade é importante como instrumento de orientação para a terapêutica inicial, bem como para o estabelecimento de protocolos de ação das equipes de saúde e comissões de controle de infecções hospitalares.

Faz-se necessário ainda uma pesquisa acerca da sensibilidade dos microrganismos aos fármacos mais utilizados, visto que o mesmo esquema antimicrobiano foi utilizado em quase metade dos casos. Com isso, seria possível, uma prescrição mais assertiva, diminuindo o sofrimento humano e a morbimortalidade resultante das infecções. Sugere-se também estudos mais acurados a respeito das pneumonias associadas à ventilação mecânica, que tragam cálculos de densidade de sua incidência e da taxa de utilização de ventilação mecânica, possibilitando, assim, um panorama mais fidedigno.

O presente estudo pode contribuir para o conhecimento dos dados epidemiológicos da IH dentro do contexto hospitalar da instituição pesquisada, possibilitando a equipe de saúde elencar estratégicas e medidas de prevenção com maior especificidade e direcionamento voltado à realidade local. Além disso, estudos como esse impulsionam e trazem notoriedade para um tema tão importante.

\section{Referências}

Barbosa, J. C. de S., Lobato, P. S., Menezes, S. A. F. de, Menezes, T. O. de A., \& Pinheiro, H. H. C. (2010). Perfil dos pacientes sob terapia intensiva com pneumonia nosocomial: principais agentes etiológicos. Revista de Odontologia UNESP, 39(4), 201-206.

Brasil. Agência Nacional de Vigilância Sanitária (2017). Critérios Diagnósticos de Infecções Relacionadas à Assistência à Saúde. Brasília: ANVISA.

Bush, K., \& Bradford, P. A. (2020). Epidemiology of $\beta$-Lactamase-Producing Pathogens. Clinical Microbiology Reviews, 33(2). doi: 10.1128/CMR.00047-19

Calcagnotto, L., Nespolo, C. R., \& Stedile, N. L. R. (2011). Resistência antimicrobiana em microrganismos isolados do trato respiratório de pacientes internados em unidade de terapia intensiva. Arquivos Catarinenses de Medicina, 40(3), 77-83.

Gaspar, M. D. R., Busato, C. R., \& Severo, E. (2012). Prevalência de infecções hospitalares em um hospital geral de alta complexidade no município de Ponta Grossa. Acta Scientiarum. Health Sciences, 34(1), 23-29. doi: 10.4025/actascihealthsci.v34i1.8943

Giuliano, K. K., Baker, D., \& Quinn, B. (2018). The epidemiology of nonventilator hospital-acquired pneumonia in the United States. American Journal of Infection Control, 46(3), 322-327. doi: 10.1016/j.ajic.2017.09.005 
Guimarães, A. C., Donalisio, M. R., Santiago, T. H. R., \& Freire, J. B. (2011). Óbitos associados à infecção hospitalar, ocorridos em um hospital geral de Sumaré-SP, Brasil. Revista Brasileira de Enfermagem, 64(5), 864-869. doi: 10.1590/S0034-71672011000500010

Horan, T. C., Andrus, M., \& Dudeck, M. A. (2008). CDC/NHSN surveillance definition of health care-associated infection and criteria for specific types of infections in the acute care setting. American Journal of Infection Control, 36(5), 309-332. doi: 10.1016/j.ajic.2008.03.002

Karvouniaris, M., Makris, D., Karabekos, D., \& Zakynthinos, E. (2011). Nosocomial MRSA pneumonia: data from recent clinical trials. Reviews on Recent Clinical Trials, 6(3), 235-240. doi: 10.2174/157488711796575568

Leiser, J. J., Tognim, M. C. B., \& Bedendo, J. (2007). Infecções hospitalares em um centro de terapia intensiva de um hospital de ensino no norte do Paraná. Ciência, Cuidado e Saúde, 6(2), 181-186. doi: 10.4025/cienccuidsaude.v6i2.4149

Leistner, R., Schröder, C., Geffers, C., Breier, A.-C., Gastmeier, P., \& Behnke, M. (2015). Regional distribution of nosocomial infections due to ESBL-positive Enterobacteriaceae in Germany: data from the German National Reference Center for the Surveillance of Nosocomial Infections (KISS). Clinical Microbiology and Infection, 21(3), 255.e1-5. doi: 10.1016/j.cmi.2014.07.015

Magalhães, V. C. R., \& Soares, V. M. (2018). Análise dos mecanismos de resistência relacionados às enterobactérias com sensibilidade diminuída aos carbapenêmicos isoladas em um hospital de referência em doenças infecto-contagiosas. Revista Brasileira de Análises Clínicas, 50(3), 278-281. doi: $10.21877 / 2448-3877.201800661$

Moura, M. E. B., Campelo, S. M. de A., Brito, F. C. P. de, Batista, O. M. A., Araújo, T. M. E. de, \& Oliveira, A. D. da S. (2007). Infecção hospitalar: estudo de prevalência em um hospital público de ensino. Revista Brasileira de Enfermagem, 60(4), 416-421.

Neves, V. D., Bulgareli, J., \& Carnut, L. (2019). Infecção Hospitalar: métodos de avaliação das medidas econômicas referentes ao tratamento e a prevenção. Journal of Management \& Primary Health Care, 11(1), 10-11. doi: 10.14295/jmphc.v11isup.945

Padrão, M. da C., Monteiro, M. L., Maciel, N. R., Viana, F. F. C. F., \& Freitas, N. A. (2010). Prevalência de infecções hospitalares em unidade de terapia intensiva. Revista da Sociedade Brasileira de Clínica Médica, 8(2), 125-128.

Pang, F., Jia, X.-Q., Zhao, Q.-G., \& Zhang, Y. (2018). Factors associated to prevalence and treatment of carbapenem-resistant Enterobacteriaceae infections: a seven years retrospective study in three tertiary care hospitals. Annals of Clinical Microbiology and Antimicrobials, 17(1), 13. doi: 10.1186/s12941-018-0267-8

Papazian, L., Klompas, M., \& Luyt, C.-E. (2020). Ventilator-associated pneumonia in adults: a narrative review. Intensive Care Medicine, 46(5), 888-906. doi: $10.1007 / \mathrm{s} 00134-020-05980-0$

Portaria $\mathrm{n}^{\circ} 2616$ (12, maio, 1998). Dispõe sobre diretrizes e normas para a prevenção e o controle das infecções hospitalares. Diário Oficial da União, BrasíliaDF, 1998.

Rocha, M. L. P., \& Dias, V. L. (2015). Epidemiologia das infecções pulmonares diagnosticadas em ambiente hospitalar: um estudo retrospectivo. Revista Brasileira de Análises Clínicas, 47(4), 141-146.

Rufino, G. P., Gurgel, M. G., Pontes, T. de C., \& Freire, E. (2012). Avaliação de fatores determinantes do tempo de internação em clínica médica. Revista da Sociedade Brasileira de Clínica Médica, 10(4), 291-297.

Salluh, J. I. F., Souza-Dantas, V. C. de, Martin-Loeches, I., Lisboa, T. C., Rabello, L. S. C. F., Saad, N., \& Póvoa, P. (2019). Ventilator-associated tracheobronchitis: an update. Revista Brasileira de Terapia Intensiva, 31(4), 541-547. doi: 10.5935/0103-507X.20190079

Santos, C. M. dos, Padula, M. P. C., \& Waters, C. (2019). Fatores de risco e incidência de Pneumonia Hospitalar em Unidade de Internação. Brazilian Journal of Health Review, 2(5), 4866-4875. doi: 10.34117/bjhr2n5-083

Santos, R. P. dos, Mariano, L. R., Takahashi, L. D. S., \& Erdmann, M. de F. (2014). Prevalência de infecção hospitalar em unidade de terapia intensiva: um estudo retrospectivo. Revista de Enfermagem da UFSM, 4(2), 410-418. doi: 10.5902/2179769211233

Silva, T. M. F. da, Cipriano Filho, A. M. A., Sousa, Z. S., Rodrigues, J. A. L., Silva, N. V. F. da, Freitas Filho, M. M. de, Pereira, J. R. G., Araújo, S. M. S. P. Rodrigues, S. T. B., \& Silva, B. N. da. (2021). Infecções hospitalares associadas à bacilos gram-negativos não fermentadores em unidade de terapia intensiva: revisão narrativa. Revista Eletrônica Acervo Saúde, 13(3), e6685. doi: 10.25248/reas.e6685.2021

Sinésio, M. C. T., Magro, M. C. S., Carneiro, T. A., \& Silva, K. G. N. da. (2018). Fatores de risco às infecções relacionadas à assistência em unidades de terapia intensiva. Cogitare Enfermagem, 23(2), e53826. doi: 10.5380/ce.v23i2.53826

Starzyk-Łuszcz, K., Zielonka, T. M., Jakubik, J., \& Życińska, K. (2017). Mortality Due to Nosocomial Infection with Klebsiella pneumoniae ESBL. Advances in Experimental Medicine and Biology, 1022, 19-26. doi: 10.1007/5584_2017_38

Turner, N. A., Sharma-Kuinkel, B. K., Maskarinec, S. A., Eichenberger, E. M., Shah, P. P., Carugati, M., Holland, T. L., \& Fowler, V. G. J. (2019). Methicillinresistant Staphylococcus aureus: an overview of basic and clinical research. Nature Reviews. Microbiology, 17(4), 203-218. doi: 10.1038/s41579-018-0147-4

Zaragoza, R., Ramírez, P., \& López-Pueyo, M. J. (2014). Infección nosocomial en las unidades de cuidados intensivos. Enfermedades Infecciosas y Microbiologia Clinica, 32(5), 320-327. doi: 10.1016/j.eimc.2014.02.006 